\title{
Research and Application of Large Data in Management Information System
}

\author{
Guo Xin \\ School of Software Service Outsourcing \\ Jishou University \\ Zhangjiajie, China \\ jianghai079@126.com
}

\begin{abstract}
With the continuous innovation and application of the modern information technology, the enterprise information is officially entered into the era of big data. And in this context, the enterprise management information system in the data search, access, storage, analysis and presentation, and put forward more challenges. In the construction of modern management information system, enterprises should pay great attention to the research and application of large data, to build a modern management information system with the times, change the traditional ideas, reform the traditional technology. In the face of challenges, enterprises need to take more effective measures to make full use of big data to create value, change the challenges as opportunities. This paper will be based on the status quo of enterprise development, the enterprise information management information system in the large data application analysis and research.
\end{abstract}

Keywords- Management information system; big data; application; data search; data storage

\section{INTRODUCTION}

Along with the advent of the information age, the information system's gradual increase in the development of information system has brought great convenience to the development and daily operation, and strengthen the management efficiency of the enterprise ${ }^{[1]}$. However, based on the big data era, enterprises in the management of information system in the data is also increasing, leading to a number of problems. First, unstructured data management, in enterprise management information system is ignored, compared with structured data management, with the development of large data, the value of unstructured data is more prominent, second, enterprise management information system is a "data island" phenomenon $^{[2-3]}$, a large number of data is lack of effective connection and integration, in the process of data management, resulting in a waste of resources. At present, many enterprise internal management information system's basic functions, are reflected in the analysis of a small amount of data, search, storage and display, the lack of large data management, is not conducive to promoting the efficient development of enterprises. Thus, in the modern big data stage, the enterprise management information system needs to adopt the unified big data management and control method ${ }^{[4]}$, the enterprise structure and the non structured data carries on the unified standard collection and storage, completes the big data application search, analysis $^{[5]}$.

\section{ANALYSIS OF LARGE DATA CHARACTERISTICS OF MODERN ENTERPRISE}

\section{A. analysis object}

According to modern enterprise management information system's internal business activities analysis, large data format is divided into two kinds of structured and unstructured data. The former refers to data in the existing fixed field, unified format and can be based on the type of relationship defined data. These data can be very good in the management information system of management; while the latter belongs to no fixed method are classified, the frequent change of the format of data, there is a data type with the characteristics of meaning and is easy to be ignored ${ }^{[6]}$. Objectively speaking, there are many characteristics of the non structured data, such as diversification, large data volume and wide distribution, and the large data collection, storage and analysis of this kind of management information system put forward higher standards.

\section{B. the significance of non structured data analysis}

Although there is no direct role in the daily business activities of the enterprise, the rational analysis of this data can produce more value indirectly. For example, through the analysis and collation of unstructured data, can objectively present the enterprise current management state, but also can be a user feedback and state of understanding, and master the behavior of enterprise employees, etc.. In the role of these information collection, more conducive to enterprise management or business decision making ${ }^{[7]}$.

Throughout some of the non structural data, most of the enterprise management information system of key data content, such as user access to enterprise web site, viewing enterprise product information, etc., through the observation and analysis of these data can help companies to understand the situation of sales products, brand influence and other key information, and then make more accurate decision. However, for the application of this kind of non structural data, most enterprises have not been attached importance to.

\section{ANALYSIS OF ENTERPRISE STRUCTURE CAPABILITY} OF LARGE DATA SYSTEM

\section{A. Standard collection of large data}

From the big data of enterprise management information system, with the increase of time will continue 
to bring more value for the development of enterprises, but also brings a lot of challenges. In particular, the standardization of large data collection work, will face the following problems: first, compatibility. Big data application module is to deal with a variety of data sources, so in the management of information system, the need to adopt a common, compatibility of the acquisition module for storage and processing; second, value. Large data applications need to maintain the value of the information, to avoid the collection of large amounts of data, there are too many failure data; third, consistency. Enterprise management information system, including the management of structural and non structural data, in the acquisition process, need to absorb different formats of data, to maintain the consistency of data acquisition processing, is conducive to the standardization of management.

In summary, the enterprise management information system under the large data standardization collection, the need to have a distributed deployment capabilities. In particular, the distribution of the acquisition module, it should be in the management information system of the various links, access to the data after the unification of the standard module for processing ${ }^{[8-9]}$. At the same time, different modules should be able to filter the data, reduce the failure and garbage collection, improve the efficiency of large data applications. Standardized data collection is to ensure the high efficiency of the management of large data applications, the need to build a universal data model, which covers data content, type, data source name or IP address, as well as data generation time and other factors.

\section{B. Large data storage}

After the acquisition of large data, the need to properly store the information management, for the search and analysis services after the search. Data storage needs to be analyzed from three perspectives: first, the actual content of the big data itself; second is the standard of large data information, which is to meet the type, time, source and content of the four elements of information content, to carry out the storage; third, the index of large data storage, for future large data applications. From a technical point of view, the enterprise management information system of large data storage, there is a certain difficulty. Because of the application of big data, not only requires the data to be wide, accurate, but also has a certain value ${ }^{[10]}$. Conventional large data storage means of upgrading, you can use the hardware hard disk. Store information data module in different hard disk partition, and let more data to backup, to ensure that data will not be easily lost, improve reliability. In addition, but also through the search ability and full text index function, to ensure that the storage of information, can be used for large data analysis to do a good job of basic protection. Set the search function, to include all and local matching, but also to include the forward and backward matching, matching data with different position and feature. Through the ambiguity of the search settings, more conducive to improving the effectiveness of data storage, reliability and utilization.

\section{PRePARE Your PAPER Before Styling}

Before you begin to format your paper, first write and save the content as a separate text file. Keep your text and graphic files separate until after the text has been formatted and styled. Do not use hard tabs, and limit use of hard returns to only one return at the end of a paragraph. Do not add any kind of pagination anywhere in the paper. Do not number text heads-the template will do that for you.

Finally, complete content and organizational editing before formatting. Please take note of the following items when proofreading spelling and grammar:

\section{A. Abbreviations and Acronyms}

Define abbreviations and acronyms the first time they are used in the text, even after they have been defined in the abstract. Abbreviations such as IEEE, SI, MKS, CGS, sc, dc, and rms do not have to be defined. Do not use abbreviations in the title or heads unless they are unavoidable.

\section{B. Analysis object}

C/S (Client/Server - PB - Client / server) or B/S (Browser/Server - Browser / server) application is only able to cope with the actual requirements of the data, the application is difficult to adapt to real needs, so we must take with the reality. Second, in the case of large data, we first have to address the growing use of information technology. Third. Data from unstructured data. Energy Internal platform for power and unification. Individual management information system has been unable to meet the needs of large data processing, and only has the functions of storage, collection, search, analysis, and early warning and trend study and other functions of the unified internal big data platform, to be able to show the best management effect.

\section{Equations}

In the RGB color model, in order to complete the color information of the image, R, G and B three color components can not be missing. According to the color moment correlation theory, the color information can be concentrated in the low order moment of the image color, the color of the color, the standard deviation and the pitch and so on. And for each color channel mean, standard deviation and inclination can will the ith channel of the $j$-th pixel PIJ represents, and $\mathrm{N}$ is the number of total image pixels, is the ith channel mean (EI), standard deviation (sigma I) and gradient (TI) respectively using the formula described as [1-3]:

$$
\begin{aligned}
& \mathbf{E}_{i}=\frac{1}{N} \sum_{j=1}^{N} P_{i j} \\
& \sigma_{i}=\left[\frac{1}{N} \sum_{\mathrm{j}=1}^{N}\left(P_{i j}-E_{i}\right)^{2}\right]^{\frac{1}{2}} \\
& t_{i}=\left[\frac{1}{N} \sum_{\mathrm{j}=1}^{N}\left(P_{i j}-E_{i}\right)^{3}\right]^{\frac{1}{3}}
\end{aligned}
$$

The 3 formulas can be used to form the HIS color model, because it has no color information, and can not be considered in the color processing, and $\mathrm{S}$ and $\mathrm{H}$ components and the human eye color. In this case, the $\mathrm{S}$ component is also able to determine. In this case, it is necessary to consider the color of the coal particle, which is effective in reducing the size of the image processing and the content of the $\mathrm{H}$. In the gray image, the gray value is the most important parameter of the coal particle image 
recognition. It is generally believed that the density of coal particles and the greater the ash, then the coal particle image will be darker, and vice versa. Thus, the characteristics of the first three order matrix can be used to describe the property. Texture features are a global feature compared to other parameters, and the nature of the food surface in the image and image region can be described. This feature is not based on the pixel features, it includes a lot of pixels in the region, and to carry out statistical calculations to get the appropriate data. And in the pattern matching, this kind of regional characteristic is more advantageous than the traditional way of comparison, it will not be because of the partial deviation which lead to the problem which cannot be matched. As a statistical feature, texture features are not deformed in their own rotation, but also can resist strong noise and high stability. To extract the texture features, the following parameters are required, i.e. gray level co-occurrence matrix, TAMURA matrix, wavelet transformation, and auto regressive texture model.

\section{Figures and Tables}

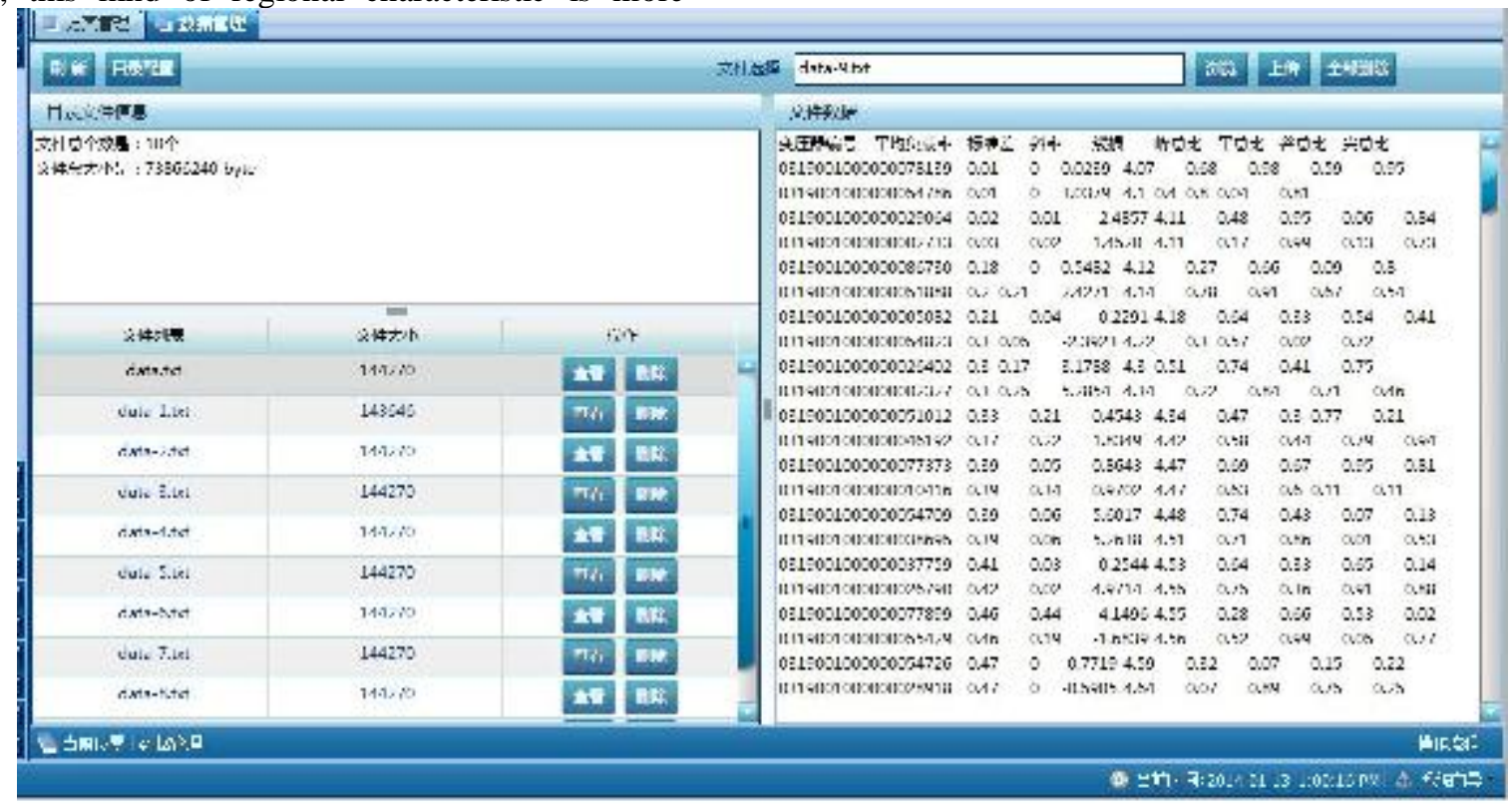

Figure 1. Large data reference data

Management information system of large data applications, the fundamental purpose is to analyze the future trend, as well as the role of decision-making, decision warning. The so-called trend analysis, that is based on a series of large data applications (collection, storage, analysis and search, etc.), to track some of the characteristics of large data, build large data samples and summarize the regularity. In the future, once the regularity changes, it can be obtained through the management of large data applications, decision-making tips, to help companies to the path of sustainable and healthy development. For example, through the use of large data applications for your enterprise user login information, you can build a model of user login information. The management information system can feedback the information, and the potential password is cracked or malicious attack website. From the current management information system big data applications, the future based on the construction of big data platform, learning model and tracking model will have a higher value, can become a key link in large data applications.

\section{CONCLUSIONS}

With the continuous innovation and development of Internet of things and cloud computing technology, the modern data explosion, the arrival of the era of big data. For the enterprise management information system, the research and application of large data has a large value, which is conducive to provide information reference for enterprise management and decision-making, and promote the sustainable and healthy development of enterprises. In the construction of modern management information system, enterprises should pay great attention to the research and application of large data, to build a modern management information system with the times, change the traditional ideas, reform the traditional technology. Through the analysis of the large data research and application of enterprise management information system, this paper summarizes the application module function and construction mode of large data in management information system, and also expounds the application direction of large data in the future.

\section{ACKNOWLEDGMENT}

A Project Supported by Scientific Research Fund of Hunan Provincial Education Department.

Project number: 14B143.

\section{REFERENCES}

[1] Guo warships. Big data. study construction of Jilin University management relations Hengtai securities company, 2015.

[2] M. Young, The Technical Writer's Handbook. Mill Valley, CA: University Science, 1989.

[3] Electronic Publication: Di gital Object Identifiers (DOIs).

[4] Zhao Chuanshen,etc. "Frequent Subtree Mining Based on Projected Branch." Journal of Computer Reseach and Development, $2006: 456 \sim 462$. 
[5] Meng Xiaofeng, kindly. "Big data management: concept, technology and challenge. " computer research and development, 2013,01:146-169.

[6] Xiong Pai Qin, Wang will give, Li Furong, Li Cuiping, Chen Hong, Zhou Xuan in Wang Shan. "Data management technology of new pattern. " Journal of software, 2013,02:175-197.

[7] Li, Liu Pengfei. "Data analysis and innovation of construction project management information system." science and technology innovation and application, 2015,18:15-16.

[8] Dong Xiaotao. "The database design of management information system. " science and technology wind, 2015,02:266.
[9] H. Goto, Y. Hasegawa, and M. Tanaka, "Efficient Scheduling Focusing on the Duality of MPL Representatives," Proc. IEEE Symp. Computational Intelligence in Scheduling (SCIS 07), IEEE Press, Dec. 2007, pp. 57-64, doi:10.1109/SCIS.2007.357670.

[10] Y. Yorozu, M. Hirano, K. Oka, and Y. Tagawa, "Electron spectroscopy studies on magneto-optical media and plastic substrate interface," IEEE Transl. J. Magn. Japan, vol. 2, pp. 740-741, August 1987 [Digests 9th Annual Conf. Magnetics Japan, p. 301, 1982]. 